\title{
Unraveling Spatio-temporal Dynamics in fMRI Recordings Using Complex ICA
}

\author{
Jörn Anemüller ${ }^{1,2}$, Jeng-Ren Duann ${ }^{1,2}$, Terrence J. Sejnowski ${ }^{1,2}$, and Scott Makeig ${ }^{1,2}$ \\ 1 Swartz Center for Computational Neuroscience, Institute for Neural Computation, \\ University of California San Diego, La Jolla, California \\ 2 Computational Neurobiology Laboratory, \\ The Salk Institute for Biological Studies, La Jolla, California, and \\ Howard Hughes Medical Institute
}

\begin{abstract}
Independent component analysis (ICA) of functional magnetic resonance imaging (fMRI) data is commonly carried out under the assumption that each source may be represented as a spatially fixed pattern of activation, which leads to the instantaneous mixing model. To allow modeling patterns of spatiotemporal dynamics, in particular, the flow of oxygenated blood, we have developed a convolutive ICA approach: spatial complex ICA applied to frequencydomain fMRI data. In several frequency-bands, we identify components pertaining to activity in primary visual cortex (V1) and blood supply vessels. One such component, obtained in the $0.10-\mathrm{Hz}$ band, is analyzed in detail and found to likely reflect flow of oxygenated blood in $\mathrm{V} 1^{1}$.
\end{abstract}

\section{Introduction}

The blood oxygenation level dependent (BOLD) contrast measured by fMRI recordings depends on the change in level of oxygenated blood with neural activity. ICA has been successful at finding independent spatial components that vary in time [1], but there may also be spatio-temporally dynamic patterns in fMRI recordings of brain activity.

Convolutive models are a way to account for dynamic flow patterns. In convolutive models, each source process is characterized by the spatio-temporal pattern it elicits and by the time-course of activation of this pattern. The signal accounted for by each source process is obtained by convolving the spatio-temporal source pattern with its time-course of activation. The mixed (measured) data are obtained by summing over the contributions of all source processes. Separation of mixed activity generated by several of such processes is not possible for instantaneous ICA algorithms since the convolutive mixing is beyond the scope of their instantaneous mixing assumption.

The convolutive separation problem can be solved by performing all computations in the frequency-domain since the convolution in the time-domain factorizes into a multiplication in the frequency-domain. Separation is performed by applying a complex ICA algorithm to the complex-valued data in each frequency-band.

The use of this procedure for the analysis of electroencephalographic (EEG) data has recently been presented elsewhere [2]. Here, we present the application of the

\footnotetext{
${ }^{1}$ Supported by the German Research Council DFG (J. A.), and by the Swartz Foundation.
} 
method to fMRI data. Compared to EEG data, fMRI data are characterized by their high spatial resolution at a low temporal sampling rate. fMRI data are commonly analyzed by spatial ICA decomposition, where time-points correspond to input dimensions and voxels to samples. This is in contrast to temporal ICA used for EEG, where sensors constitute input dimensions and time-points samples. To apply complex ICA to fMRI signals, we analogously apply spatial complex ICA to frequency-domain fMRI data.

\section{Methods}

Consider measured signals $x_{t i}$, where $t$ denotes time and $i$ denotes voxels. Their spectral time-frequency representations $x_{T i}(f)$ are computed using the short-term Fourier transformation

$$
x_{T i}(f)=\sum_{\tau} x_{i}(T+\tau) h(\tau) e^{-\mathfrak{i} 2 \pi f \tau / 2 K}
$$

where $f$ denotes center frequency, and $h(\tau)$ is a Hanning window. Hence, data of size [times $t \times$ voxels $i$ ] are transformed into data of size [times $T \times$ voxels $i \times$ frequencies $f$ ].

For each frequency band $f$, the signals are modeled to be generated from independent sources $s_{T i}(f)$ by multiplication with frequency-specific mixing coefficients $a_{T T^{\prime}}(f)$,

$$
x_{T i}(f)=\sum_{T^{\prime}} a_{T T^{\prime}}(f) s_{T^{\prime} i}(f),
$$

which in matrix notation reads

$$
\mathbf{X}(f)=\mathbf{A}(f) \mathbf{S}(f) .
$$

Complex ICA is used to obtain the independent components using the linear projection

$$
\mathbf{U}(f)=\mathbf{W}(f) \mathbf{X}(f)
$$

where $u_{T i}(f)$ and $w_{T T^{\prime}}(f)$ represent complex spatial component patterns and the separating matrix, respectively.

Hence, as in real-valued ICA for fMRI signals, a spatial ICA decomposition is performed, where time-points correspond to input dimensions and voxels to samples. For each frequency-band, we obtain a set of complex-valued independent components, each characterized by its associated complex time-course $\mathbf{a}_{T^{\prime}}(f)$ and complex-valued spatial pattern $\mathbf{s}_{T^{\prime}}(f)$, with $T^{\prime}$ denoting component number.

The complex infomax ICA algorithm $[2,3]$ is a generalization of the real-valued infomax ICA algorithm [4] and models the sources as complex random variables with a circular symmetric, super-Gaussian probability density function. Analysis of the statistics of frequency-domain fMRI data strongly indicates that these assumptions are fulfilled. Matrices $\mathbf{W}(f)$ are found using natural gradient optimization [5]

$$
\tilde{\nabla} \mathbf{W}(f)=\nabla \mathbf{W}(f) \mathbf{W}(f)^{H} \mathbf{W}(f)=\left(\mathbf{I}-\mathbf{V}(f) \mathbf{U}(f)^{H}\right) \mathbf{W}(f),
$$

where $\mathbf{V}(f)$ is a non-linear function of the source estimates $\mathbf{U}(f)$ :

$$
\begin{aligned}
& v_{T i}(f)=\operatorname{sign}\left(u_{T i}(f)\right) g\left(\left|u_{T i}(f)\right|\right), \\
& \operatorname{sign}(z)= \begin{cases}0 & \text { if } z=0, \\
z /|z| & \text { if } z \neq 0 .\end{cases}
\end{aligned}
$$



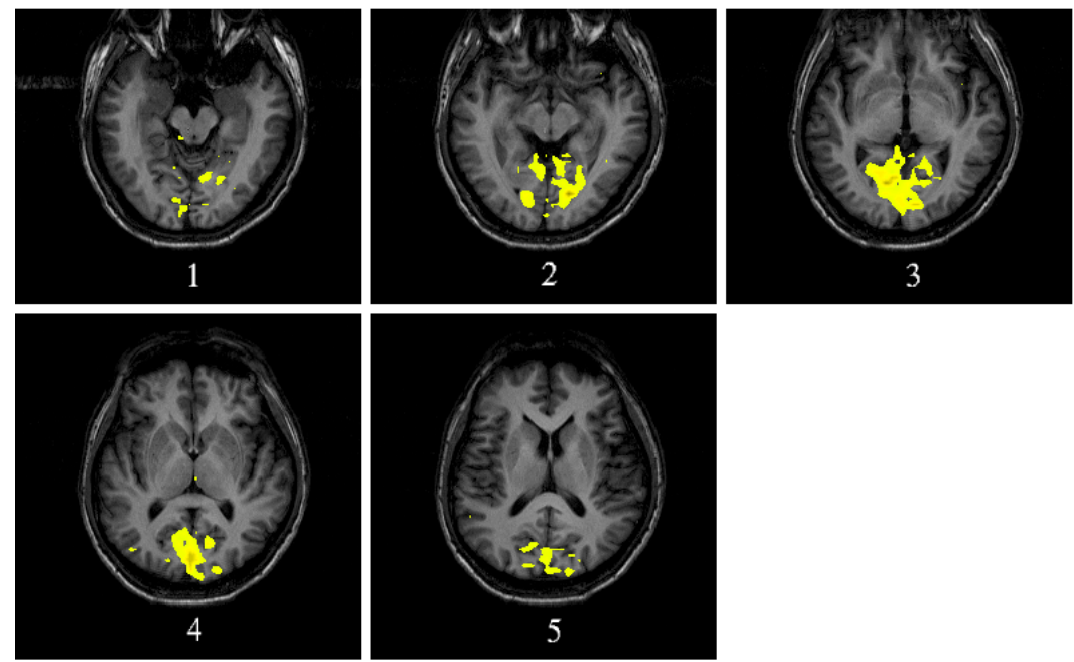

Fig. 1. Magnitude map of the component region of activity (ROA) for complex component IC2 obtained by complex ICA in the $0.1-\mathrm{Hz}$ frequency-band. The ROA extends over visual area V1 and blood supply vessels. Light yellow colors indicate component magnitude in ROA. Structural image of the recorded areas is plotted in darker gray tones. The component ROA is interpolated to the higher resolution of the structural scan for better visualization. (Note: The electronic version of this document contains color figures for better visualization and can be obtained from the first author).

I denotes the identity matrix and the function $g(\cdot): \mathbb{R} \rightarrow \mathbb{R}$ is a real-valued non-linearity, chosen as $g(x)=\tanh (x)$.

\section{Results}

The experimental data were from a $250 \mathrm{~s}$ experimental session consisting of ten epochs with stimulus onset asynchrony (SOA) of $25 \mathrm{~s}$. An 8-Hz flickering checkerboard stimulus was presented to one subject for $3.0 \mathrm{~s}$ at the beginning of each epoch. 500 timepoints of data were recorded at a sampling rate of $2 \mathrm{~Hz}(\mathrm{TR}=0.5)$ with resolution $64 \times 64 \times 5$ voxels, field-of-view $250 \times 250 \mathrm{~mm}^{2}$, slice thickness $7 \mathrm{~mm}$. Standard preprocessing included removal of off-brain and low-intensity voxels, reducing the data to 2863 voxels. For more experiment details refer to [6]. The data of this experiment are freely available as part of the FMRLAB toolbox for fMRI ICA analysis [7].

Spectral decomposition was performed using the windowed discrete Fourier transformation (1) with a Hanning window of length 40 samples, a window shift of 1 sample, and frequency-bands $0.05,0.10, \ldots, 1.00 \mathrm{~Hz}$. This resulted in data split into 20 bands, each with 461 time-points and 2863 voxels.

Spatial complex ICA decomposition was performed within each frequency-band. In a preprocessing step, input dimensionality in each band was reduced from 461 to 50 by retaining only the subspace spanned by the (complex) eigenvectors corresponding 

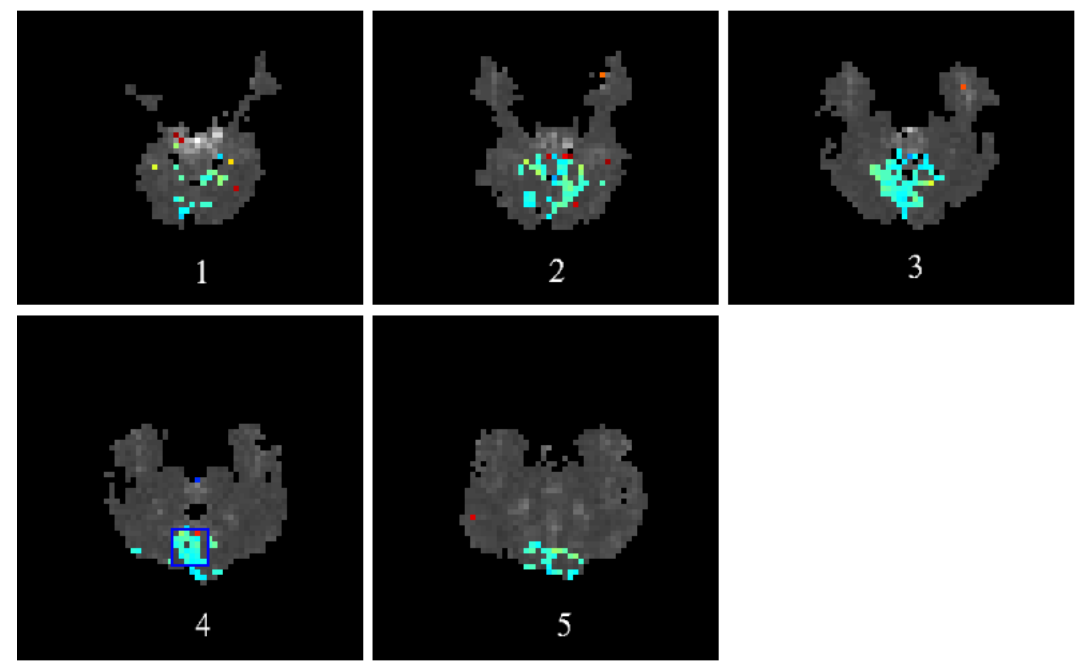

Fig. 2. Phase map of the component ROA for complex component IC2. Light green colors indicate component phase in region of activity. Note that in contrast to Fig. 1 the information is displayed at the lower spatial resolution of the functional recordings. The voxels marked by a blue square in slice 4 are investigated further in Fig. 4.

to the 50 largest eigenvalues of the data matrix $\mathbf{X}(f)$. Complex ICA decomposed this subspace into 50 complex independent components per band.

Motivated by previous results of real-valued infomax ICA on the same data [6], we were interested in components with a region of activity (ROA) near primary visual cortex V1. One such component was found in several frequency-bands, with a timecourse of activation that closely reflected the SOA of the visual stimulus. Time-locking of component activity to stimulus presentation was particularly reliable for component number 2 (IC2) in the $0.10-\mathrm{Hz}$ band. IC2 was the single clearly V1-related component in this band. The following analysis is restricted to this particular component.

Fig. 1 displays the magnitude of the complex spatial component map of IC2 in the ROA of the five recording slices. The ROA was determined from $z$-scores of the component map by transforming each component map to zero mean and unit variance, and setting a heuristic threshold of 1.5. The extent of IC2 from the centrally located main blood vessels to primary visual cortex is clearly visible, in particular in slices 3 and 4. The complex component's phase in the ROA is displayed in Fig. 2. Slices 3 and 4 display a phase shift from the upper left border of the component ROA image towards the lower right border. The phase shift indicates a time lag in the activation of the component voxels when transformed back into the time-domain which will be further investigated below. Fig. 3 shows power (squared magnitude) and phase of the component time-course of activation. Component power clearly reflects the pattern of visual stimulation with an SOA of $25 \mathrm{~s}$, with peaks in power that follow stimulation with a time lag of about $9 \mathrm{~s}$, and a high dynamic range between component activity and inactivity. The component phase regularly advances and appears to be time locked to 

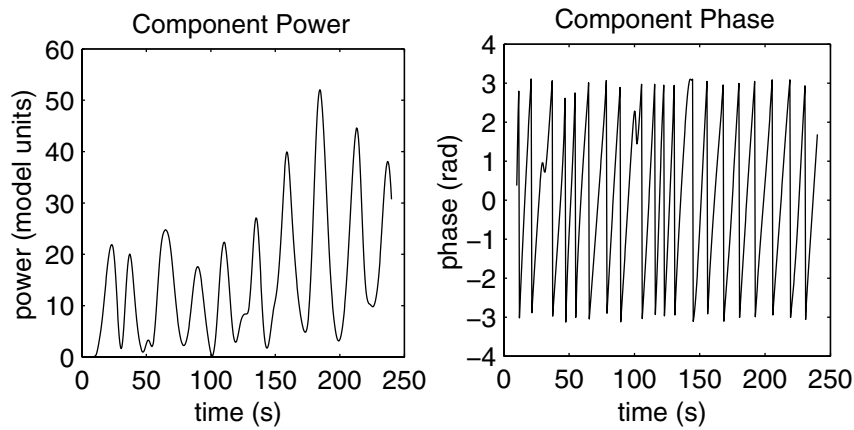

Fig. 3. Time-course of power (left) and phase (right) of complex component IC2 in the $0.1-\mathrm{Hz}$ frequency-band. Note the time-locking of amplitude and phase to stimulus presentation in $25 \mathrm{sec}-$ onds intervals. The first and last 10 seconds of the experiment are not shown because computation of the spectral components was stopped when the analysis window (length $20 \mathrm{~s}$ ) reached the edges of the recording. The time-interval from $179.5 \mathrm{~s}$ to $187.0 \mathrm{~s}$ around the largest component power peak is investigated further in figure 4.

stimulus presentation, possibly to a lower degree during the periods from $0 \mathrm{~s}$ to $50 \mathrm{~s}$ and from $100 \mathrm{~s}$ to $130 \mathrm{~s}$, which could be due to the subject's level of attention to the stimulus.

Complex voxel activity induced by the component may be obtained by backprojecting the complex time-course to the complex spatial map, i.e., by forming the product $\mathbf{a}_{T^{\prime}}(f) \mathbf{s}_{T^{\prime}}(f)$, where $T^{\prime}$ denotes component number, $\mathbf{a}_{T^{\prime}}(f)$ the corresponding column of the mixing matrix $\mathbf{A}(f)$, and $\mathbf{s}_{T^{\prime}}(f)$ the corresponding row of the source matrix $\mathbf{S}(f)$. Transforming the complex frequency-domain voxel activity to the real time-domain reduces - in the case of a window-shift of one sample and a single frequency-band - to taking the real-part. We performed these steps to analyze time-domain voxel activity induced by the component near the largest component power peak between $179.5 \mathrm{~s}$ and $187.0 \mathrm{~s}$ of the experiment. Fig. 4 displays the activity within a patch of 24 voxels located in recording slice 4, marked by a blue square in Fig. 2. Following stimulus presentation at $175.0 \mathrm{~s}$, activity in the patch started to increase with a time lag of about $4.5 \mathrm{~s}$, first in the voxels most centrally located in the brain (top row of voxels in each plot of Fig. 4), and propagating within about $1 \mathrm{~s}$ to the posterior voxels of to primary visual cortex (bottom row in each plot of Fig. 4). Analogously, voxel activity decreased first in the top row of voxels before decreasing in the bottom rows.

To investigate whether similar time lag effects can be found without ICA processing, we also computed the $0.10-\mathrm{Hz}$ band activity of the recorded data at the 24 voxels that have been investigated in Fig. 4, using the same spectral decomposition that has been used for the complex ICA decomposition. Activity accounted for by recorded data and by IC2 was separately averaged within each voxel row, starting with row 1 for the most centrally located voxels, and up to row 6 for the voxels in the posterior position. The resulting averages are plotted in Fig. 5 for recorded data and for component induced activity. Since the signals are band-limited, we obtain oscillatory activity with positive and negative swings. The analysis of relative time lags and amplitudes near the peak of 


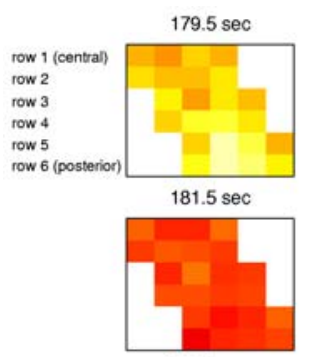

$183.5 \mathrm{sec}$

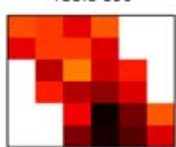

$185.5 \mathrm{sec}$

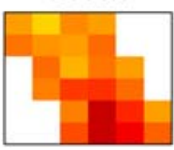

$180 \mathrm{sec}$

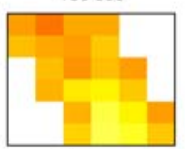

$182 \mathrm{sec}$

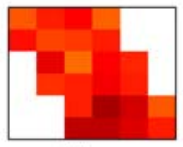

$184 \mathrm{sec}$

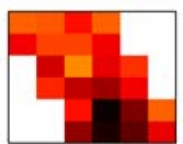

$186 \mathrm{sec}$

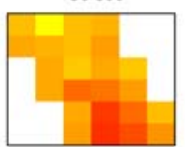

$180.5 \mathrm{sec}$

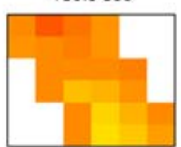

$182.5 \mathrm{sec}$

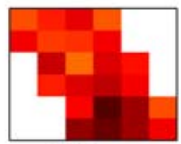

$184.5 \mathrm{sec}$

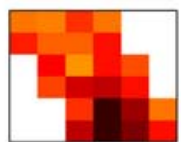

$186.5 \mathrm{sec}$

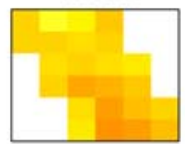

$181 \mathrm{sec}$

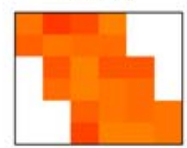

$183 \mathrm{sec}$

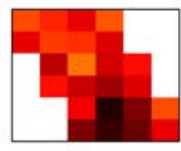

$185 \mathrm{sec}$

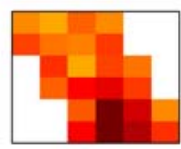

$187 \mathrm{sec}$

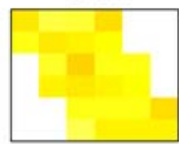

Fig. 4. Backprojected component activity from complex component IC2. Complex component time-course was backprojected to corresponding activity at the voxels and transformed to the time-domain. Shown is the activity of 24 voxels in visual area V1, the position of which is marked by a blue box in slice 4 of Fig. 2. The flickering-checkerboard stimulus was presented for $3.0 \mathrm{~s}$ at experiment time $175.0 \mathrm{~s}$ (not shown). Activation started to increase with a time lag of about $4.5 \mathrm{~s}$, with first increase occuring at the centrally-located voxels (top rows), and propagated to the posterior voxels (bottom rows) within approximately $1 \mathrm{~s}$. This is compatible with over-supplied oxygenated blood propagating in the posterior direction and being washed out through the drainage vein from area V1.

component power (at $184.5 \mathrm{~s}$ ) is not influenced by this fact. In the component induced activity, the more centrally located voxels are activated between $0.5 \mathrm{~s}$ and $1.0 \mathrm{~s}$ prior to the posterior voxels. The time lag increases monotonously with more posterior voxel position. This gradient of posterior voxels being activated later than the central voxels is also reflected in the activity of the recorded voxels signals. However, the voxels in row 3 form an exception since their extremal activation occurs even after the posterior voxels are activated. The analysis of activation amplitudes in Fig. 5 gives similar results: The component induced amplitude increases monotonously towards more posterior voxel position. Overall, this tendency is also found in the recorded signals, but some exceptions occur, e.g., amplitude in row 2 is smaller than in row 1.

\section{Discussion and Conclusion}

We analyzed fMRI signals using a convolutive ICA approach which enabled us to model patterns of spatio-temporal dynamics. Parameters for this model were efficiently estimated in the frequency-domain where the convolution factorizes into a product. Our method consists of three processing stages: 1) Computing time-frequency representations of the recorded signals, using short-term Fourier transformation. 2) Separation 

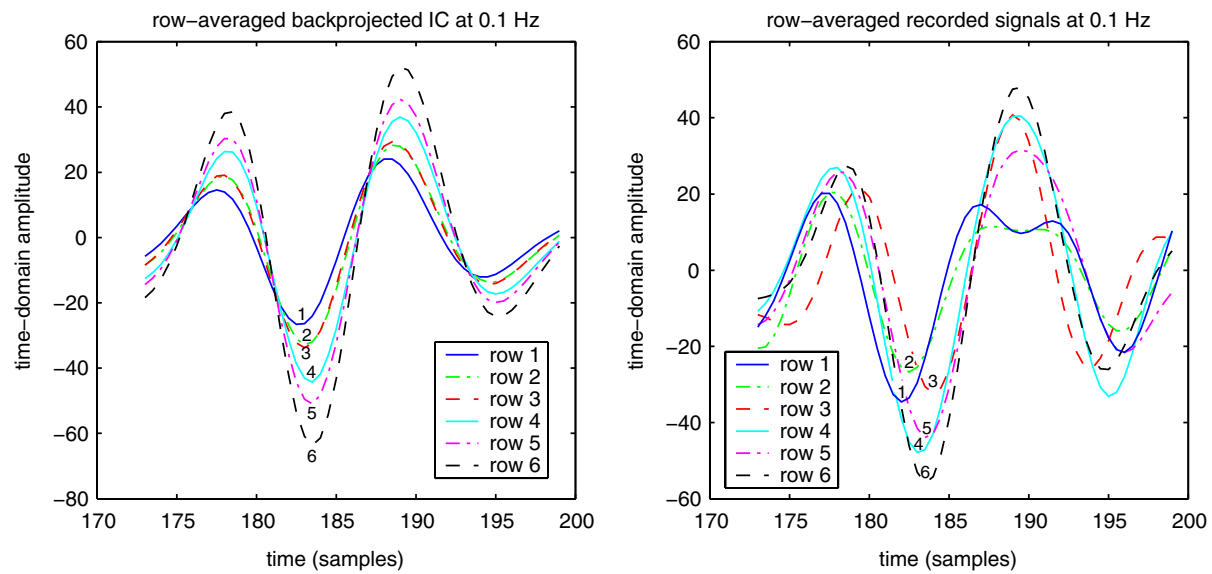

Fig. 5. Left: Average time-courses near largest component power peak (at $184.5 \mathrm{~s}$ ) for each row of $0.1-\mathrm{Hz}$ band time-domain backprojected component activations displayed in Fig. 4. Row 1 corresponds to the most centrally located voxels, row 6 to the posterior ones. Right: Corresponding average time-courses computed from the recorded activations in the $0.1-\mathrm{Hz}$ band of the same voxels. For the average IC activation (left), the voxel-rows are activated in the order $1-(2,3)-(4,5,6)$ with row 6 being activated with a time lag of about 1 second with respect to row 1 . This lag is compatible with blood supply propagating across the patch in the posterior direction. In the average recorded activations (right), the voxel-rows are activated in the order $1-2-4-(5,6)-3$. With the exception of row 3 , this also indicates a posterior direction of propagation. The most posterior voxel-row of backprojected component IC2 shows strongest activation which is plausible since it is closest to the drainage vein. The same tendency is found in the recorded signals, but ordering of amplitude of voxel-rows is not as monotonous as for IC2. Backprojected IC activations may represent a cleaner picture of the stimulus related process with respect to phase- and amplitude-gradient, because activity of other ongoing brain processes is canceled out.

of the measured signals into independent components using spatial complex infomax ICA in each frequency-band. 3) Computing the corresponding dynamic voxel activation pattern induced by each independent component in the time-domain.

From data of a visual stimulation fMRI experiment we obtained a complex component in the $0.1-\mathrm{Hz}$ band with a component map ROA extending across primary visual cortex and its blood supply vessels. By reconstructing the spatio-temporal activation pattern accounted for by this component, we identified a time lag of about $1 \mathrm{~s}$ between activation of central and posterior voxels. A related time lag, but distributed less regularly, could be observed in the $0.1-\mathrm{Hz}$ frequency-band of the measured signals. The amplitude of component-induced voxels activations increased in the posterior direction. Also this trend could be seen in the recorded signals, but it was less systematic than for the ICA processed signals.

Both observations are compatible with the physiology underlying generation of the fMRI signal. The posterior voxels in the component ROA are the ones closest to the posterior drainage vein. The convergence of over-supplied oxygenated blood towards the drainage vein may therefore result in the large amplitudes for these voxels. The 
temporal delay between activation of central and posterior voxels is consistent with the propagation of over-supplied oxygenated blood from the centrally located arteries to the posterior drainage vein. Similar temporal delays have been observed from optical recordings of intrinsic signals, related to blood oxygenation, in monkey visual cortex [8].

These results may indicate that frequency-domain complex infomax ICA can capture patterns of spatio-temporal dynamics in the data. It is reassuring that similar dynamics could also be observed in the recorded (mixed) signals, making the possibility of the complex ICA results being mere processing artifacts implausible. On the other hand, the spatio-temporal dynamics emerged with a higher degree of regularity and physiological plausibility from the complex ICA results than from the measured data. Separation of the stimulus evoked activity from interfering, ongoing brain activity by the complex ICA method appears as the natural explanation for this observation.

Here, we have focused the analysis on a single frequency-band. Taking into account information from other frequency-bands in which components have been found near V1 should allow us to reconstruct the full time-domain spatio-temporal dynamics associated with visual stimulation.

In conjunction with previous results reported on modeling the spatio-temporal dynamics in EEG signals with complex ICA [2], the results presented here are a further indication that convolutive models may be useful for analyzing a wide range of data.

\section{References}

1. M. J. McKeown, T.-P. Jung, S. Makeig, G. Brown, S. S. Kindermann, T. W. Lee, and T. J. Sejnowski. Spatially independent activity patterns in functional MRI data during the Stroop color-naming task. Proc. Natl. Acad. Sci. U. S. A., 95:803-810, 1998.

2. J. Anemüller, T. J. Sejnowski, and S. Makeig. Complex independent component analysis of frequency-domain electroencephalographic data. Neural Networks, 16:1311-1323, 2003.

3. J. Anemüller and B. Kollmeier. Adaptive separation of acoustic sources for anechoic conditions: A constrained frequency domain approach. Speech Communication, 39(1-2):79-95, Jan 2003.

4. A. J. Bell and T. J. Sejnowski. An information maximization approach to blind separation and blind deconvolution. Neural Computation, 7:1129-1159, 1995.

5. S. Amari, A. Cichocki, and H. H. Yang. A new learning algorithm for blind signal separation. In D. Touretzky, M. Mozer, and M. Hasselmo, editors, Advances in Neural Information Processing Systems 8, pages 757-763, Cambridge, MA, 1996. MIT Press.

6. Jeng-Ren Duann, Tzyy-Ping Jung, Wen-Jui Kuo, Tzu-Chen Yeh, Scott Makeig, Jen-Chuen Hsieh, and Terrence J. Sejnowski. Single-trial variability in event-related BOLD signals. NeuroImage, 15:823-835, 2002.

7. J.-R. Duann, T.-P. Jung, and S. Makeig. FMRLAB: Matlab software for independent component analysis of fMRI data. World Wide Web publication, 2003. http://sccn.ucsd.edu/fmrlab.

8. R. M. Siegel, J. R. Duann, T. P. Jung, and T. J. Sejnowski. Independent component analysis of intrinsic optical signals for gain fields in inferior parietal cortex of behaving monkey. In Society for Neuroscience Abstracts 28, 2002. 